INGENIERÍA QUÍMICA

\title{
Hidrotalcitas de NiZnFe y NiMgFe modificadas con V Y Cr como precursores de catalizadores para deshidrogenación oxidativa de propano
}

CHEMICAL ENGINEERING

\section{NiZnFe and NiMgFe hydrotalcites modified with $\mathrm{V}$ and $\mathrm{Cr}$ as precusors of catalysts for oxidative dehydrogenation of propane}

\author{
Santiago Mesa*, Johana Arboleda*, Sandra Amaya*, Adriana Echavarría* $§$ \\ *Universidad de Antioquia, Catalizadores y Adsorbentes \\ Facultad de Ciencias Exactas, Instituto de Química \\ Facultad de Ingeniería, Departamento de Ingeniería Química \\ Calle 67No.53-108, Medellín-Colombia
}

§smesae@msn.com,jocare@gmail.com,salily104@gmail.com,aechavar@udea.edu.co

(Recibido: Diciembre 15 de 2011- Aceptado: Noviembre 19 de 2012)

\begin{abstract}
Resumen
En el presente trabajo se sintetizaron dos materiales trimetálicos tipo hidrotalcita usando $\mathrm{Ni}, \mathrm{Zn}, \mathrm{Mg}$ y Fe. Posteriormente, dichos materiales se modificaron con especies de V y Cr. Los materiales obtenidos se caracterizaron por espectroscopía de absorción atómica, difracción de rayos $\mathrm{X}$, análisis termogravimétrico (ATG) y análisis térmico-diferencial (ATD). Mediante ATG se determinó una temperatura de calcinación de $500^{\circ} \mathrm{C}$ para la obtención de los óxidos mixtos los cuales se evaluaron en la reacción de deshidrogenación oxidativa de propano (DHOP) usando una velocidad espacial de $50 \mathrm{ml} / \mathrm{g}$.min , obteniéndose el mejor resultado para el sistema NiMgFe con un rendimiento del 5.13\% y una selectividad hacia el propeno del $34.35 \%$, a una temperatura de $400^{\circ} \mathrm{C}$.
\end{abstract}

Palabras clave: Deshidrogenación oxidativa, Hidrotalcitas, Óxido mixto, Propeno

\begin{abstract}
In this paper, two trimetallic hydrotalcite-like materials were synthesized using $\mathrm{Ni}, \mathrm{Zn}, \mathrm{Mg}$ and Fe. Further, those materials were modified with $\mathrm{V}$ and $\mathrm{Cr}$ species. Characterization of the obtained materials was carried out by atomic absorption spectroscopy, $\mathrm{X}$ ray diffraction, thermogravimetric analyses (TGA) and differential thermal analyses (DTA). Calcination temperature of $500^{\circ} \mathrm{C}$ was determined by TGA in order to obtain mixed oxides, which were evaluated in oxidative dehydrogenation of propane (ODHP) using a space velocity of 50 $\mathrm{ml} / \mathrm{g}$.min. The best result was obtained using NiMgFe system, with yield of $5.13 \%$ and selectivity to propene of $34.35 \%$ at temperature of $400^{\circ} \mathrm{C}$.
\end{abstract}

Keywords: Hydrotalcites, Mixed oxides, Oxidative dehydrogenation, Propylene 


\section{Introducción}

Los materiales tipo hidrotalcita, también conocidos como hidróxidos dobles laminares (HDLs), son un grupo de materiales objeto de muchas investigaciones debido al gran número de aplicaciones que tienen a nivel catalítico (oxidación parcial y reformado con vapor de hidrocarburos, síntesis de metanol, reacciones de metilación vía Fischer Tropsch), Gabriele et al. (2008). Los HDLs presentan una estructura tipo brucita, $\operatorname{Mg}(\mathrm{OH})_{2}$, donde el catión $\mathrm{Mg}^{2+}$ se encuentra coordinado octaédricamente con seis aniones $\mathrm{OH}^{-}$y los diferentes octaedros comparten aristas, formando láminas infinitas. Cuando los iones $\mathrm{Mg}^{2+}$ son parcialmente sustituidos por iones trivalentes, se genera un exceso de carga positiva la cual es compensada por un anión que se ubica entre dos láminas tipo brucita, Kovanda et al. (2005).

La fórmula general de los materiales tipo hidrotalcita es: $\mathrm{M}_{1-x}^{\mathrm{II}} \mathrm{M}^{\mathrm{III}}(\mathrm{OH})_{2}\left(\mathrm{~A}^{\mathrm{n}-}\right)_{x / \mathrm{n}} \cdot y \mathrm{H}_{2} \mathrm{O}$, donde $\mathrm{M}^{\mathrm{II}}$ y $\mathrm{M}^{\mathrm{III}}$ son los iones divalentes $\mathrm{y}$ trivalentes, respectivamente; $\mathrm{A}^{\mathrm{n}-}$ es el anión de compensación de carga, $y$ es el número de moles de agua, y $x$ representa la fracción molar del catión trivalente, Cavani et al. (1991).

Estudios realizados, Roman Bulánek et al. (2012), Lin Wang et al. (2012), Gheorghita Mitran et al. (2012), han demostrado que los metales de transición tipo redox, son promisorios en la deshidrogenación oxidativa de propano. Una de la rutas para obtener catalizadores de óxidos metálicos con diferentes composiciones es a través de tratamiento térmico de precursores laminares, que tras el colapso de sus laminas, dan lugar a la formación de óxidos mixtos porosos (catalizadores). La ventaja de este método de obtención de catalizadores es que permite modificar fácilmente sus propiedades tales como área superficial, carácter ácido-base, y sustitución de elementos o compuestos con relativa facilidad, todo esto mediante la síntesis del precursor. En este sentido, los materiales tipo hidrotalcita han sido usados principalmente como precursores de catalizadores debido a las interesantes propiedades de los óxidos obtenidos luego de calcinación: gran área superficial, propiedades básicas, formación de óxidos mixtos homogéneos y el llamado "efecto memoria", el cual permite la reconstrucción de la estructura hidrotalcita cuando el material es sometido a una solución acuosa que contiene varios aniones, Cavani et al. (1991). En general, los materiales tipo hidrotalcita pueden ser transformados en óxidos multimetálicos (óxidos mixtos) mediante calcinación a temperaturas entre $400-500^{\circ} \mathrm{C}$. Dado que el colapso de la estructura laminar ocurre a temperaturas menores de $500^{\circ} \mathrm{C}$, entonces la difusión de iones metálicos durante esta etapa puede ser ignorada. Por ende, los óxidos obtenidos suelen adquirir una alta dispersión catiónica a partir de los materiales tipo hidrotalcita, Auerbach et al. (2004). Los catalizadores obtenidos a partir de materiales tipo hidrotalcitas han sido objeto de investigación para diferentes reacciones, tales como hidrogenación, deshidrogenación, isomerización y esterificación. Rives et al. (1998) estudiaron la hidrogenación del acetileno a etileno con óxidos mixtos preparados a partir de hidrotalcitas de $\mathrm{ZnNiAlCr}$, encontrando que la presencia de zinc inhibía la formación de coque y el incremento en el contenido de níquel generaba una disminución en la actividad. Por otro lado, se ha encontrado que las propiedades redox del níquel favorecen la actividad y selectividad en reacciones de hidrogenación, principalmente; y la presencia del $\mathrm{ZnO}$ disminuye la formación de coque, Auerbach et al. (2004). Asimismo, varios estudios sobre deshidrogenación de alcanos (propano y etilbenceno, principalmente) usando materiales tipo hidrotalcitas como precursores de catalizadores han sido reportados en la literatura, Malherbe et al. (1998), Ye et al. (2004), Crivello et al. (2005), Mitchell et al. (2005), Valverde et al. (2012). La deshidrogenación de propano a propeno ha sido llevada a cabo en presencia de $\mathrm{CO}_{2}$ usando un catalizador tipo hidrotalcita de $\mathrm{CrMgAl}$, para el cual se encontraron sitios ácidos y básicos, responsables de la actividad catalítica, Auerbach et al. (2004). Del mismo modo, catalizadores derivados de hidrotalcitas de $\mathrm{ZnFeAl}$ han sido desarrollados para la deshidrogenación de etilbenceno a estireno, donde la máxima actividad catalítica fue alcanzada con una relación $\mathrm{Fe}: \mathrm{Zn}: \mathrm{Al}$ igual a 1:6:2, respectivamente; Auerbach et al. (2004). En cuanto a la modificación de hidrotalcitas, se ha logrado principalmente mediante la incorporación de un anión metálico de interés dentro de la zona interlaminar, 
lo cual puede presentar ventajas respecto a una hidrotalcita no modificada dentro de una reacción. Específicamente para la reacción de deshidrogenación oxidativa de propano (DHOP), se ha usado principalmente el vanadio y el molibdeno como metales de interés. Dula et al. (2002) lograron la incorporación de especies de vanadio en una hidrotalcita de $\mathrm{MgAl}$ para su posterior estudio en la reacción de DHOP en un intervalo de temperaturas de 550 a $800^{\circ} \mathrm{C}$, obteniendo selectividades hacia propeno entre el 25 y el $75 \%$. Particularmente, el desarrollo de nuevos catalizadores para la reacción de deshidrogenación oxidativa de propano ha suscitado un especial interés, debido a las limitaciones termodinámicas que presentan las rutas convencionales para la obtención de olefinas, Cavani et al. (2007). De acuerdo a esto, en el presente trabajo se sintetizaron dos materiales tipo hidrotalcita, basados en NiZnFe y NiMgFe, los cuales fueron posteriormente modificados con aniones de $\mathrm{V}$ y $\mathrm{Cr}$; los seis materiales obtenidos (modificados y $\sin$ modificar) sirvieron como precursores de los catalizadores, finalmente evaluados en la reacción de deshidrogenación oxidativa de propano en un rango de temperatura de 200 a $500^{\circ} \mathrm{C}$ con una velocidad espacial (Vs) de 50 $\mathrm{ml} / \mathrm{g} . \mathrm{min}$.

\section{Metodología}

\subsection{Síntesis}

Se prepararon dos precursores trimetálicos $\mathrm{Ni} /$ $\mathrm{Zn} / \mathrm{Fe}$ y $\mathrm{Ni} / \mathrm{Mg} / \mathrm{Fe}$ con las siguientes relaciones molares en el gel: $\mathrm{Fe}\left(\mathrm{NO}_{3}\right)_{3}: 1.5 \mathrm{Zn}\left(\mathrm{NO}_{3}\right)_{2}: 1$. $5 \mathrm{Ni}\left(\mathrm{NO}_{3}\right)_{2}: 2.1 \mathrm{Na}_{2} \mathrm{CO}_{3}: 6.8 \mathrm{NaOH}: 380.8 \mathrm{H}_{2} \mathrm{O}$ y $\quad \mathrm{Fe}\left(\mathrm{NO}_{3}\right)_{3}: 1.5 \mathrm{Mg}\left(\mathrm{NO}_{3}\right)_{2}: 1.5 \mathrm{Ni}\left(\mathrm{NO}_{3}\right)_{2}: 2.1 \mathrm{~N}$ $\mathrm{a}_{2} \mathrm{CO}_{3}: 6.8 \mathrm{NaOH}: 383.7 \mathrm{H}_{2} \mathrm{O}$ respectivamente, como se describe a continuación: el precursor llamado $\mathrm{NiMgFe}$ se preparó a partir de dos soluciones acuosas dispuestas por separado, una conteniendo $\mathrm{Ni}\left(\mathrm{NO}_{3}\right)_{2} \cdot 6 \mathrm{H}_{2} \mathrm{O}$ (Merck), $\mathrm{Mg}\left(\mathrm{NO}_{3}\right)_{2} \cdot 6 \mathrm{H}_{2} \mathrm{O}$ (Merck) y $\mathrm{Fe}\left(\mathrm{NO}_{3}\right)_{3} \cdot 9 \mathrm{H}_{2} \mathrm{O}$ (Merck), la cual se denominó solución $\mathrm{A}$, mientras que la otra conteniendo $\mathrm{Na}_{2} \mathrm{CO}_{3}$ (Merck) y $\mathrm{NaOH}$ (Merck), solución B. La solución B es adicionada gota a gota a la solución A. El gel resultante se dejó en agitación durante media hora para luego llevarlo a la estufa dentro de un reactor de acero inoxidable con recubrimiento interno de teflón durante 24 horas a $130^{\circ} \mathrm{C}$; luego, el sólido formado se lavó y se recuperó por filtración. De manera similar se sintetizó el material $\mathrm{NiZnFe}$, sustituyendo la fuente de $\mathrm{Mg}$ por una de $\mathrm{Zn}\left(\mathrm{Zn}\left(\mathrm{NO}_{3}\right)_{2} \cdot 4 \mathrm{H}_{2} \mathrm{O}\right.$, Merck) con temperatura y tiempo de cristalización de $80^{\circ} \mathrm{C}$ y $48 \mathrm{~h}$, respectivamente.

\subsection{Modificación}

La modificación de los materiales se realizó vía intercambio iónico siguiendo el siguiente procedimiento: se preparó una solución de dicromato de potasio $\left(\mathrm{K}_{2} \mathrm{Cr}_{2} \mathrm{O}_{7}\right.$, Riedel-de Haën) o de vanadato de amonio ( $\mathrm{NH}_{4} \mathrm{VO}_{3}$, Merck) en agua. Luego, se adicionó el sólido previamente sintetizado y sin calcinar a la solución de dicromato o vanadato (según sea el caso). La suspensión resultante se dejó en agitación durante 24 horas a temperatura ambiente. Luego de pasado este tiempo el sólido se recuperó por filtración y se secó. La relación molar $\mathrm{M} / \mathrm{CO}_{3}{ }^{-2}$, donde $\mathrm{M}$ : $\mathrm{VO}_{3}^{-}$ó $\mathrm{Cr}_{2} \mathrm{O}_{7}^{-2}$, se mantuvo entre 4 y 5 .

Los sólidos obtenidos, modificados y sin modificar, se sometieron a un tratamiento térmico para dar lugar a la formación de óxidos mixtos. Este proceso se llevó a cabo en una mufla Fischer Scientific Isotemp.

\subsection{Caracterización}

Los sólidos se caracterizaron por difracción de rayos $\mathrm{X}$ en un difractómetro Rigaku, usando una fuente de radiación de cobre con longitud de onda $\lambda=1.5418 \AA$, en un intervalo de $3-40^{\circ}$ en dos theta, a una velocidad de paso de $2 \% \mathrm{~min}$. Los análisis térmicos se efectuaron en un equipo TA Instruments Hi-Res ATG 2950 y ATD 1600, en atmósfera de nitrógeno en un intervalo de temperatura de 30 a $800^{\circ} \mathrm{C}$ con una velocidad de calentamiento de $10^{\circ} \mathrm{C} / \mathrm{min}$. El análisis químico en los sólidos se determinó por espectroscopía de absorción atómica en un equipo iCE3000.

\subsection{Catálisis}

El análisis de los productos de reacción se realizó en un cromatógrafo SHIMADZU GC9A, conectado en línea al sistema de reacción y utilizando columnas Molecular Sieve 5A (MS) 
y Porapack Q (PQ). La reacción se llevó a cabo en un reactor de cuarzo alimentado con propano al $98 \%$ y aire seco, con una relación molar de la mezcla reaccionante propano/oxígeno de 2:1. Se trabajó a temperaturas entre 200 y $500^{\circ} \mathrm{C}$, una velocidad espacial de $50 \mathrm{ml} / \mathrm{g} \cdot \mathrm{min}$, y $0.4226 \mathrm{~g}$ de catalizador. Para cada corrida, la conversión se calculó a partir de los picos de cada producto de reacción y a partir del propano sin reaccionar, usando los factores de respuesta determinados experimentalmente. La selectividad se calculó con el área del pico del propeno dividido entre la suma de las áreas de todos los productos corregidos con el factor de respuesta.

\section{Resultados y discusión}

\subsection{Caracterización}

Los difractogramas para los precursores $\mathrm{NiMgFe}$ y NiZnFe se muestran en la figura 1. Se observa que los dos patrones de difracción sugieren una semejanza estructural con la fase hidrotalcita, obtenida a partir de la base de datos PDF-4 (01-089-0460). Del mismo modo, para ambos materiales sintetizados, se observa un pequeño desplazamiento hacia ángulos menores en $2 \theta$ de las reflexiones correspondientes a la fase hidrotalcita, siendo mayor el desplazamiento para el material $\mathrm{NiMgFe}$. El desplazamiento observado en la posición de las señales es atribuido a la diferente composición de los materiales sintetizados en este trabajo con respecto al patrón simulado en la base de datos PDF-4.

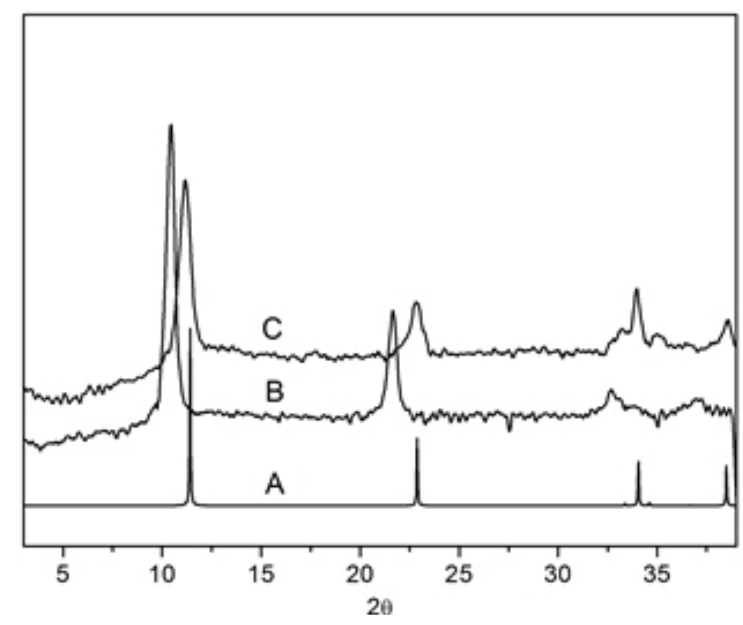

Figura 1. Difractogramas de (A) MgAl Teórico $\mathrm{PDF}-4$, (B) NiMgFe y (C) NiZnFe
De igual forma, en las figuras 2 y 3 se muestran los análisis de DRX realizados a los materiales luego de intercambiar con cromo y vanadio para ambos sistemas, NiMgFe y NiZnFe. Se puede observar que ambos materiales siguen manteniendo su estructura laminar luego del intercambio. Sin embargo, los materiales modificados presentaron menor definición en las señales, exhibiendo una menor relación señal/ ruido que los materiales sin modificar. Esto puede deberse a una baja en la cristalinidad ya que es muy difícil obtener una fase altamente cristalina luego de realizar un proceso de modificación, siendo más factible una degeneración de la estructura por la presencia de este nuevo anión.

Por otro lado, el material NiZnFe modificado con $\mathrm{Cr}(\mathrm{NiZnFe}-\mathrm{Cr})$, si bien presenta las señales características de la fase hidrotalcita, también presenta señales inusuales entre 25 y $32.5^{\circ}$ en $2 \theta$ (Figura 2), lo que podría indicar impurezas en el material, las cuales no pudieron ser identificadas. Esta anomalía probablemente fue causada por el pH ácido de la solución de sal de cromo en la que se realizó el intercambio. Los medios ácidos son especialmente "agresivos" con este tipo de sólidos, pudiendo causar incluso lixiviación de las láminas tipo brucita.

A partir del contenido de $\mathrm{Ni}, \mathrm{Zn}, \mathrm{Mg}$ y $\mathrm{Fe}$ obtenidos por análisis químicos y compuestos volátiles $\mathrm{H}_{2} \mathrm{O}$ y $\mathrm{CO}_{2}$ obtenidos por análisis térmicos, se logró determinar la fórmula química para cada uno de los materiales como:

$\left[\mathrm{Ni}_{0.41} \mathrm{Mg}_{0.48} \mathrm{Fe}_{0.09}\right](\mathrm{OH})_{2}\left(\mathrm{CO}_{3}\right)_{0.04} \cdot \mathrm{H}_{2} \mathrm{O}$ y
$\left[\mathrm{Ni}_{0.45} \mathrm{Zn}_{0.44} \mathrm{Fe}_{0.09}\right](\mathrm{OH})_{2}\left(\mathrm{CO}_{3}\right)_{0.05} \cdot 0.8 \mathrm{H}_{2} \mathrm{O}$

Basándose en la fórmula general para materiales tipo hidrotalcita. En la tabla 1 se comparan los resultados obtenidos experimentalmente y los teóricos calculados a partir de la fórmula química propuesta.

Del mismo modo, usando espectroscopía de absorción atómica, se evidenció la presencia de especies de cromo y vanadio en los materiales, la cual causó degeneración en la estructura tipo hidrotalcita como se evidenció en los análisis de DRX. En la tabla 2 se observan los resultados mediante análisis elemental. 


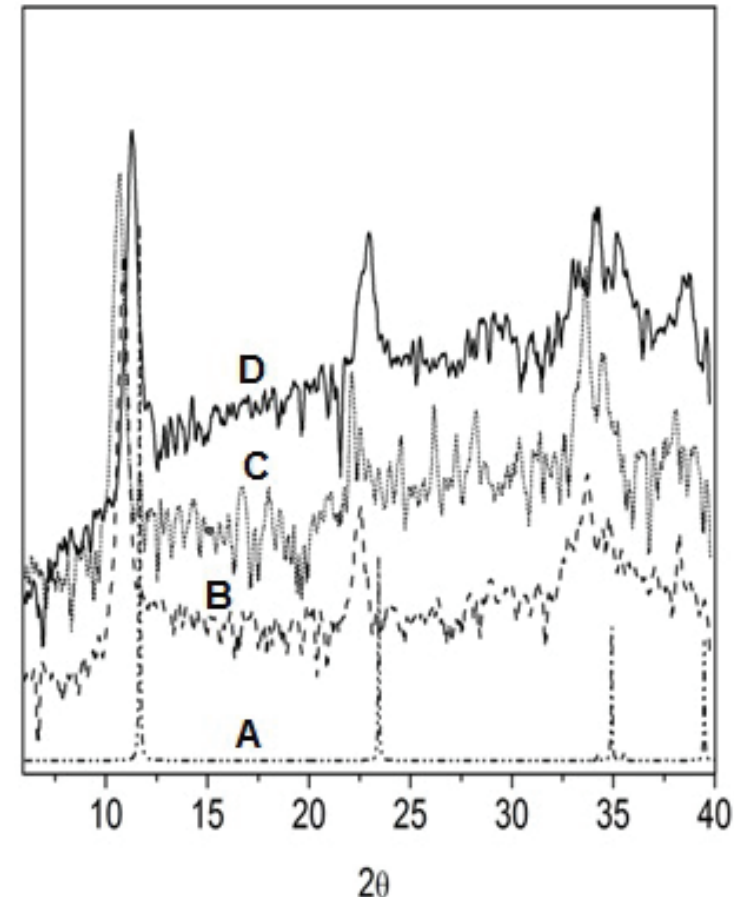

Figura 2. Difractogramas de los materiales modificados en el sistema NiZnFe (A)MgAl Teórico PDF-4, (B)NiZnFe sin modificar, (C)NiZnFe-Cr y (D) $\mathrm{NiZnFe}-\mathrm{V}$

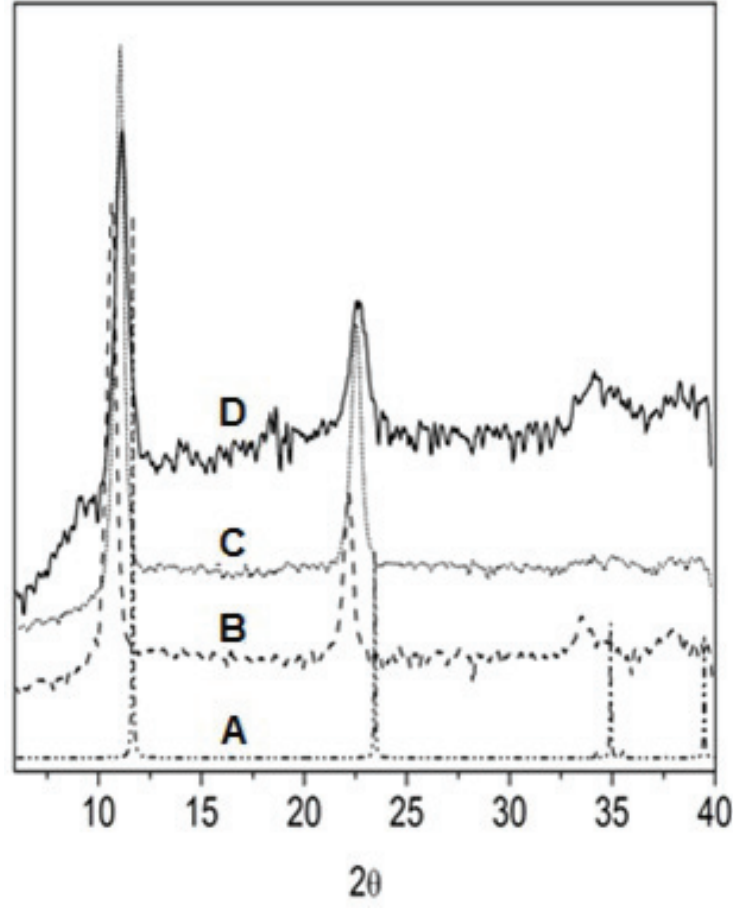

Figura 3. Difractogramas de los materiales modificados en el sistema $\mathrm{NiMgFe} \mathrm{(A)} \mathrm{MgAl} \mathrm{Teórico}$ $\mathrm{PDF}_{-4}$, (B) NiMgFe sin modificar, (C) NiMgFe-Cry (D) $\mathrm{NiMgFe}-\mathrm{V}$

Tabla 1. Composición teórica y experimental.

\begin{tabular}{ccccccc}
\hline \multirow{2}{*}{ Muestra } & \multicolumn{3}{c}{ \%Elemento (absorción atómica) } & \multicolumn{2}{c}{ \%Volátiles (ATG) } \\
& $\mathrm{Mg}$ & $\mathrm{Zn}$ & $\mathrm{Ni}$ & $\mathrm{Fe}$ & $\mathrm{CO}_{2}$ & $\mathrm{H}_{2} \mathrm{O}$ \\
\hline $\mathrm{NiMgFe}$ & $11.07(12.28)^{*}$ & ---- & $23.01(25.52)$ & $4.85(5.38)$ & $2.12(2.13)$ & $15.37(18.67)$ \\
$\mathrm{NiZnFe}$ & ---- & $23.78(25.87)$ & $21.82(23.73)$ & $4.46(4.85)$ & $2(1.91)$ & $11.06(12.77)$ \\
\hline
\end{tabular}

*Valores teóricos en paréntesis

Tabla 2. Contenido de Vy Cr en los materiales modificados.

\begin{tabular}{ccc}
\hline \multirow{2}{*}{ Muestra } & \multicolumn{2}{c}{ \% Elemento } \\
& $\mathrm{V}$ & $\mathrm{Cr}$ \\
\hline NiMgFe-V & 1.23 & ---- \\
NiMgFe-Cr & ---- & 2.15 \\
NiZnFe-V & 1.69 & ---- \\
NiZnFe-Cr & ---- & 6.25 \\
\hline
\end{tabular}

Observando los análisis térmicos mostrados en la figura 4, se pueden observar tres eventos marcados para los materiales sin modificar. El primer evento corresponde a la pérdida de agua físicamente adsorbida y agua interlaminar, el segundo evento se le atribuye a la deshidroxilación de las láminas tipo brucita, y por último, el tercer evento corresponde a la deshidroxilación total y eliminación de los aniones presentes en la región interlaminar, entre ellos el carbonato. Del mismo modo, las diferencias en los termogramas de ambos materiales son muy pequeñas, como se puede observar en la figura 4, lo que significa que las diferencias en la composición de material volátil es insignificante, como se comprobó mediante la fórmula propuesta para dichos sólidos. Igualmente, los análisis térmicos realizados a los materiales modificados (Figura 5) presentaron la misma tendencia que los sólidos sin modificar sin embargo se observa una evidente diferencia en pérdidas de peso entre los sólidos modificados y sin modificar, lo que puede indicar una sustitución parcial del carbonato por especies no volátiles en la zona interlaminar, debido a la dificultad de evitar la contaminación de especies carbonato presentes 
en el ambiente y a la afinidad de esta especie por incorporarse en la zona interlaminar, Auerbach et al. (2004). Esta diferencia en pérdidas de peso se evidencia aún más con el material $\mathrm{NiZnFe}$ modificado con $\mathrm{Cr}$, sin embargo dicha diferencia, bien sea para los materiales modificados con Vó

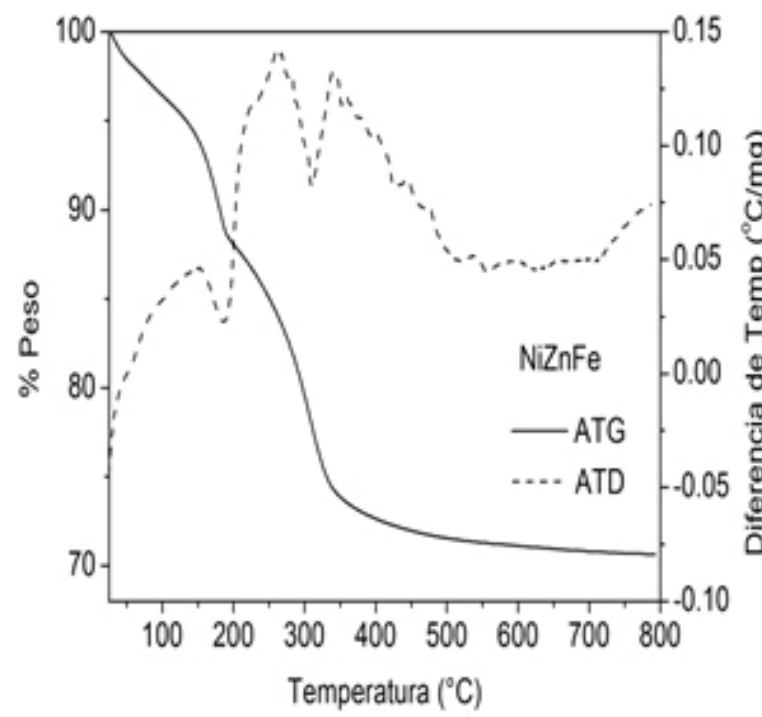

Cr, no es coincidente con los análisis químicos, cuyos resultados arrojaron valores mayores que ésas diferencias encontradas por ATG. Debido a esto se cree que ocurrió una pobre intercalación mientras que en su mayoría se llevó a cabo una impregnación sobre la superficie del sólido.

Figura 4. Análisis térmicos de (a) NiZnFe y (b) NiMgFe

Asimismo, a partir de los análisis térmicos realizados se evidenció que los materiales alcanzaron la estabilidad térmica alrededor de los $500^{\circ} \mathrm{C}$. Por ello se decidió calcinar los precursores a dicha temperatura para garantizar la formación completa de los óxidos correspondientes. Una vez calcinados, los catalizadores resultantes se caracterizaron por DRX, presentando difractogramas poco definidos lo que da cuenta de una pobre cristalinidad, con algunas señales débiles características de óxidos metálicos correspondientes a cada material, identificados en la base de datos PDF-4 como FeO (00-0011223), NiO (00-001-1239), $\mathrm{MgO}$ (00-0011235), $\mathrm{ZnO}$ (01-070-2551). Los catalizadores modificados con $\mathrm{Cr}$ y V presentaron patrones de difracción muy similares a los correspondientes catalizadores sin modificar. Esto es atribuido a que la relación entre la cantidad de óxidos metálicos de $\mathrm{Ni}, \mathrm{Zn}, \mathrm{Mg}$ y $\mathrm{Fe}$ y la cantidad de compuestos de $\mathrm{V}$ o $\mathrm{Cr}$ es muy alta, lo que ocasiona que no se puedan apreciar las señales correspondientes a dichos compuestos. Sin embargo, esto no significa que los compuestos formados a partir de $\mathrm{V}$ o Cr no estén presentes en los catalizadores. En la figura 6 solo se muestran los difractogramas de los catalizadores sin modificar.

\subsection{Catálisis}

A partir de los estudios catalíticos realizados, se evidenció que los catalizadores fueron activos en la reacción de deshidrogenación oxidativa de propano. El mejor desempeño catalítico para el sistema NiZnFe sin modificar se alcanzó a $350^{\circ} \mathrm{C}$, con una conversión de propano del $12.04 \%$ y selectividad del $17.35 \%$ hacia el propeno. Además, se presentó una alta selectividad hacia $\mathrm{CO}_{2}$ correspondiente al $82.65 \%$. Mientras que para el sistema NiMgFe sin modificar, se logró una selectividad hacia propeno de alrededor del $35 \%$ y hacia $\mathrm{CO}_{2}$ del $64 \%$ a una temperatura de $400^{\circ} \mathrm{C}$ (Figura 7).

De igual forma, para ambos catalizadores se observó un descenso en la actividad catalítica a temperaturas superiores a $400^{\circ} \mathrm{C}$, donde se observó su desactivación, siendo altamente selectivos hacia $\mathrm{CO}_{2}$ con conversiones casi completas de $\mathrm{O}_{2}$. 

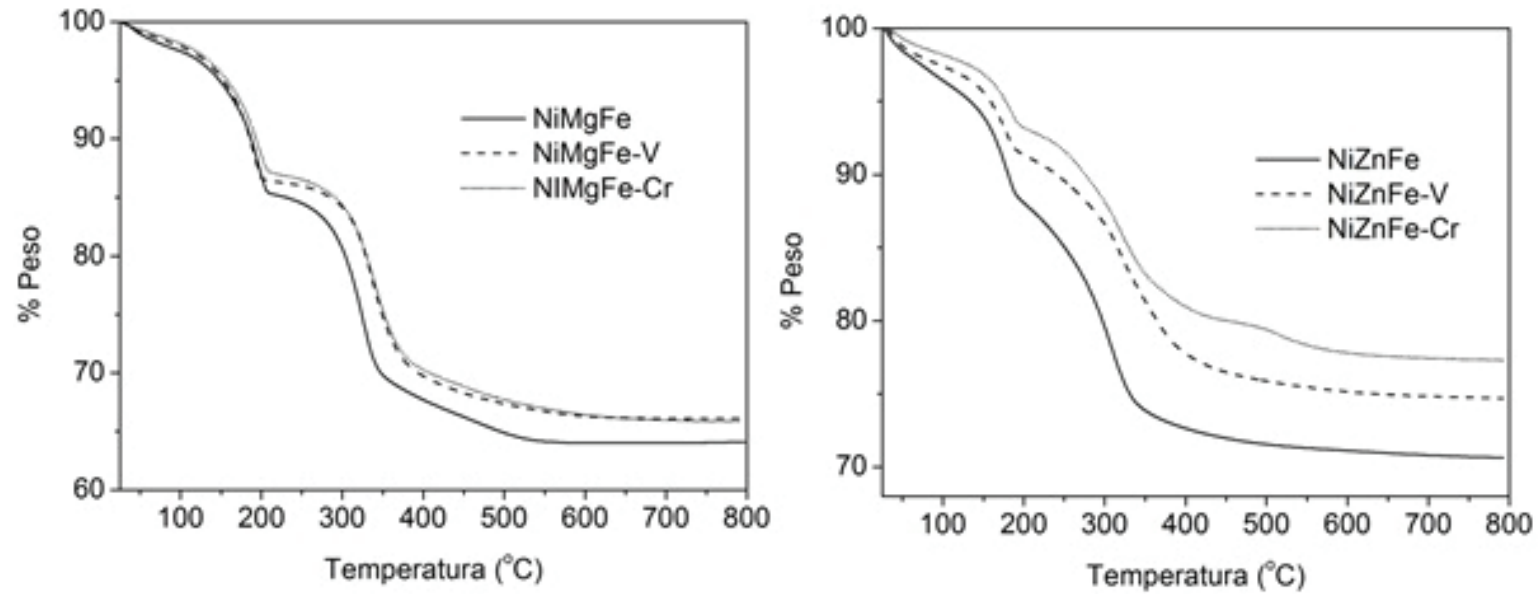

Figura 5. Análisis térmicos de los materiales modificados
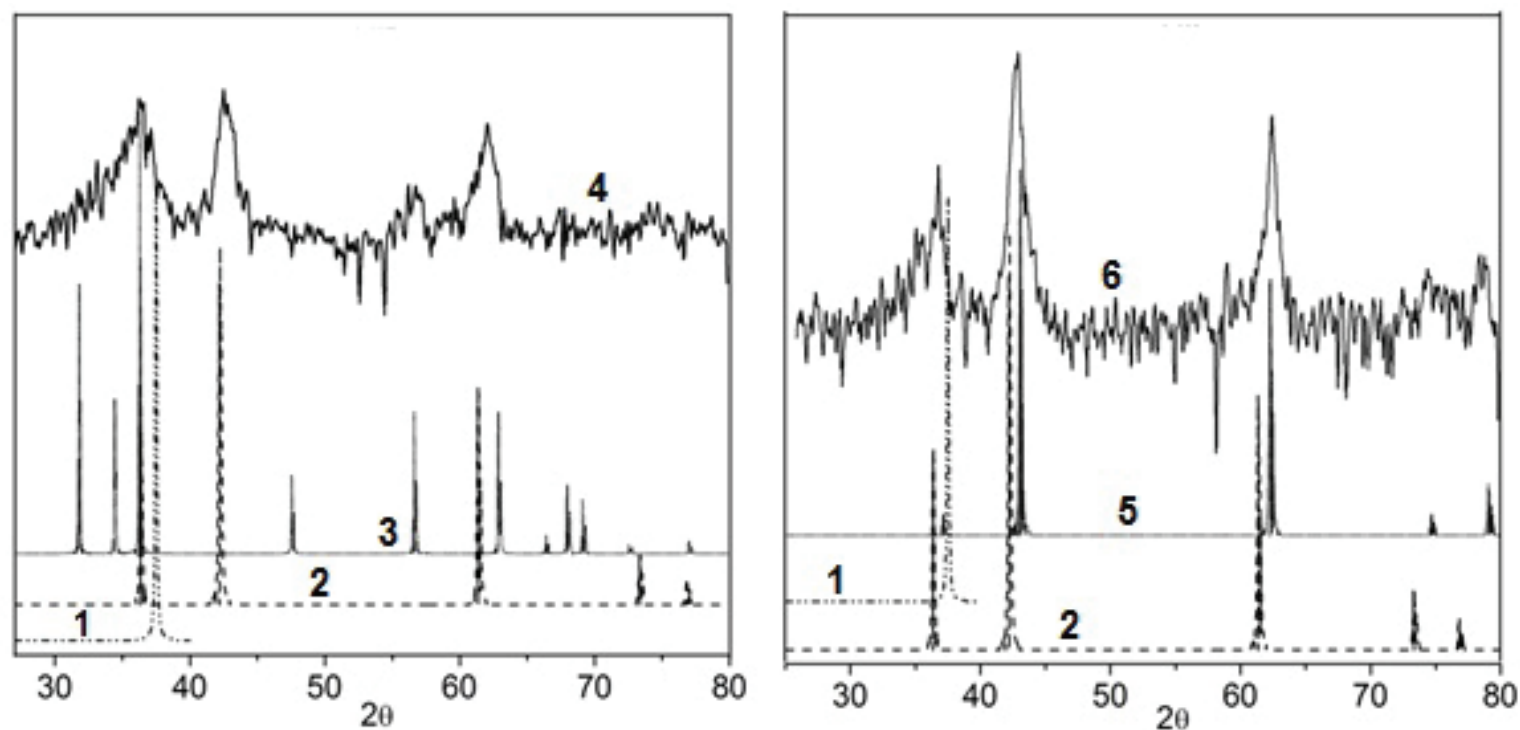

Figura 6. Difractogramas de los catalizadores sin modificar NiMgFe (6) y NiZnFe (4), y de los posibles óxidos formados $\mathrm{NiO}$ (1), $\mathrm{FeO}$ (2), $\mathrm{ZnO}$ (3) y $\mathrm{MgO}$ (5).
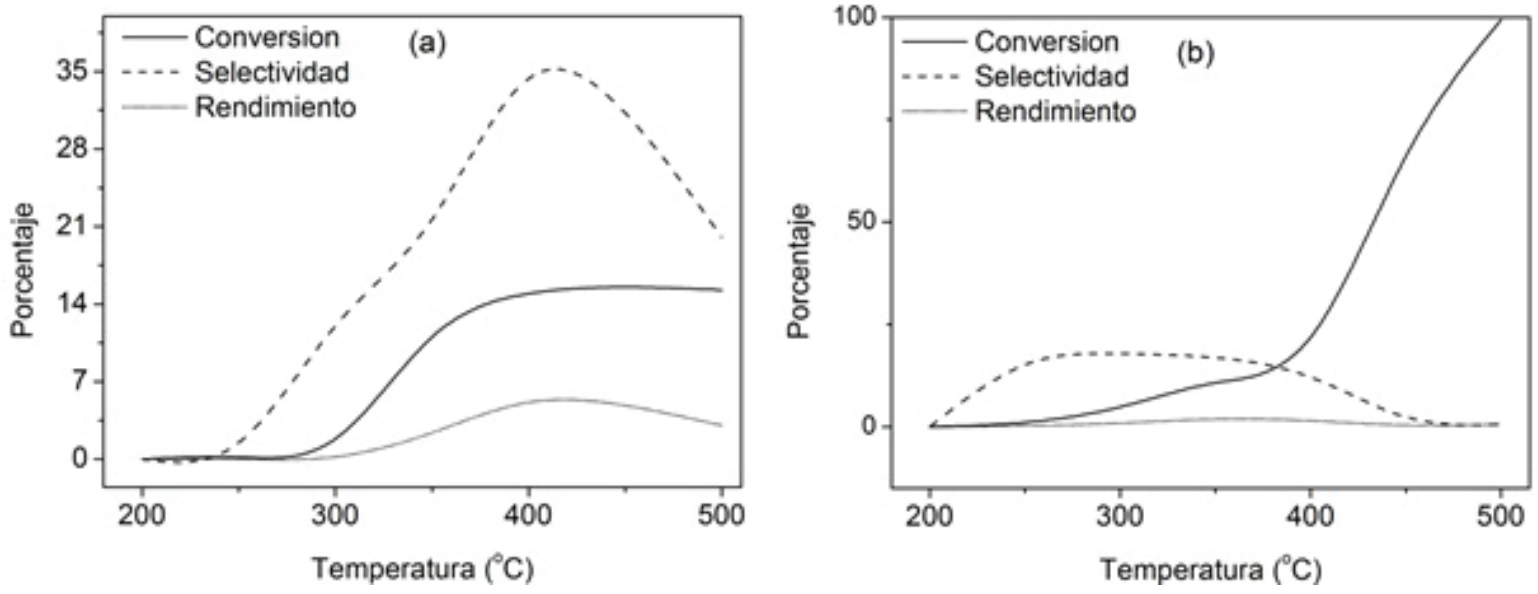

Figura 7. Resultados catalíticos para (a) NiMgFec500 y (b) NiZnFec500 a velocidad espacial de $50 \mathrm{ml} / \mathrm{g} . \mathrm{min}$ 
En la figura 8 se presentan los resultados de conversión de propano y selectividad hacia el propeno de los catalizadores modificados con Cry V del sistema NiMgFe y su correspondiente sin modifcar. Se observa que los materiales modificados, bien sea con $\mathrm{Cr}$ o $\mathrm{V}$, presentan mayor conversión que el material sin modificar, especialmente a $500^{\circ} \mathrm{C}$. Es así como se observó una conversión de alrededor del $60 \%$ para el catalizador con contenido de V. Por otro lado, para el catalizador modificado con $\mathrm{Cr}$ la conversión fue de aproximadamente $92 \%$ a $500^{\circ} \mathrm{C}$. Sin embargo, aunque la conversión

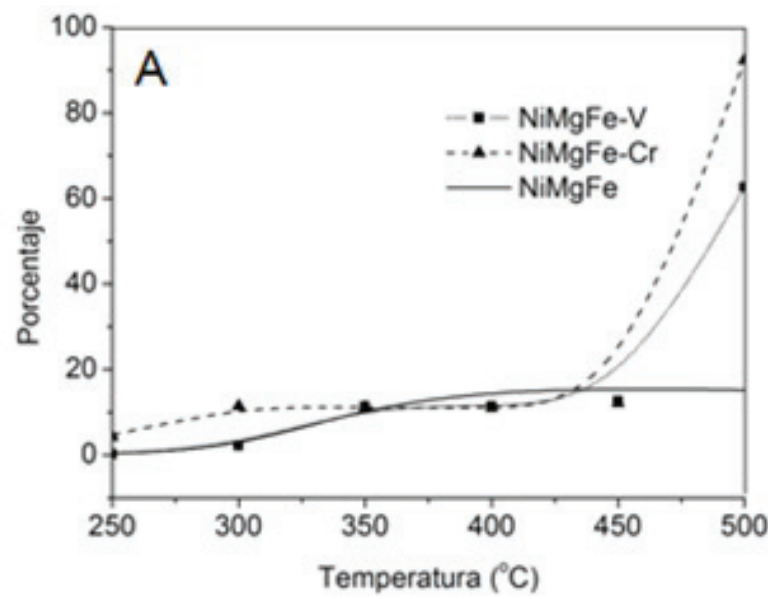

aumentó con la incorporación de especies de $\mathrm{Cr}$ y V, la selectividad hacia propeno se vio reducida en los catalizadores modificados a partir de $300^{\circ} \mathrm{C}$ en comparación con el material sin modificar, este comportamiento es algo bastante común en la deshidrogenación de alcanos de bajo peso molecular, Gheorghita Mitran et al. (2012). Además de esto, si consideramos que hay una mayor cantidad de centros activos, habrá un incremento en la posibilidad de una oxidación "preferente" del propano, y por ende una mayor conversión.

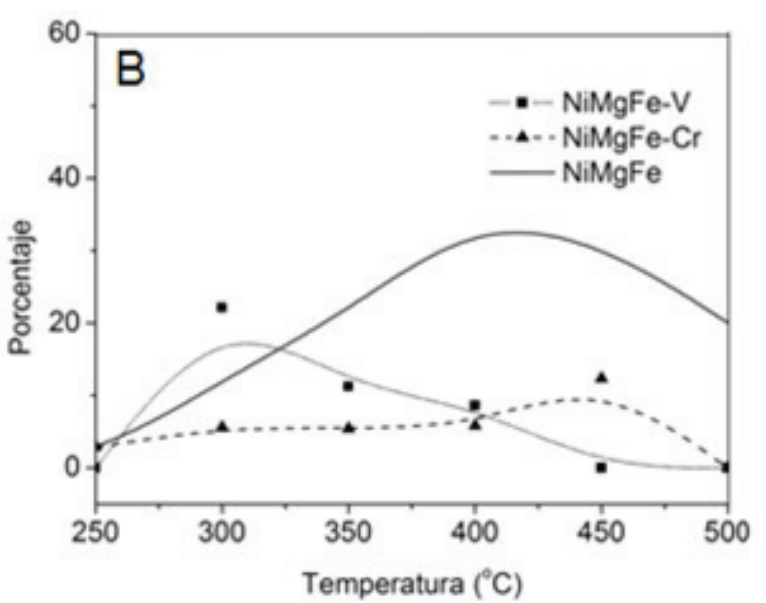

Figura 8. Resultados de (A) Conversión y (B) Selectividad de los materiales en el sistema NiMgFe

También se evidenció durante el estudio catalítico, que la incorporación de $\mathrm{Cr}$ en el material $\mathrm{NiMgFe}$ aumentó significativamente la conversión de propano a $500^{\circ} \mathrm{C}$, sin embargo, por debajo de $450^{\circ} \mathrm{C}$ los resultados de conversión fueron muy similares a los del material sin modificar. A pesar de que la incorporación de Cr aumentó la conversión, la selectividad se vio reducida en comparación con el material sin modificar. Esto sugiere que el $\mathrm{Cr}$ presente en el material podría ser un promotor de la reacción, activando el propano y haciendo que aumente la conversión.

Los catalizadores en el sistema NiZnFe (Figura 9) presentaron desactivación por sinterización, favorecida por las altas temperaturas y la presencia de vapor de agua, por encima de los $450^{\circ} \mathrm{C}$, Caballero (2002), lo que significa que la presencia de especies de $\mathrm{V}$ y $\mathrm{Cr}$ no favorecieron la estabilidad térmica a altas temperaturas como era de esperarse, dada la pobre incorporación y la alta impregnación. Sin embargo la selectividad se vio favorecida a bajas temperaturas con la incorporación de especies de $\mathrm{V}$, alcanzándose un valor de alrededor del $40 \%$ a $300^{\circ} \mathrm{C}$, mientras el catalizador con $\mathrm{Cr}$ presentó pobres resultados en conversión y selectividad, donde se observó una tendencia constante al incrementar la temperatura.

De acuerdo con los resultados catalíticos, los materiales presentaron actividad en la reacción de DHOP a bajas temperaturas (Dula et al. (2002), Mitchell et al. (2005), Cavani et al. (2007)) alrededor de $300^{\circ} \mathrm{C}$, esto puede ser atribuido a la presencia de $\mathrm{Ni}$, el cual ayuda a la activación del alcano a bajas temperaturas debido a sus propiedades redox, lo que resulta ser promisorio para la producción de olefinas de interés comercial, dada la reducción en el gasto energético y menor impacto ambiental. 

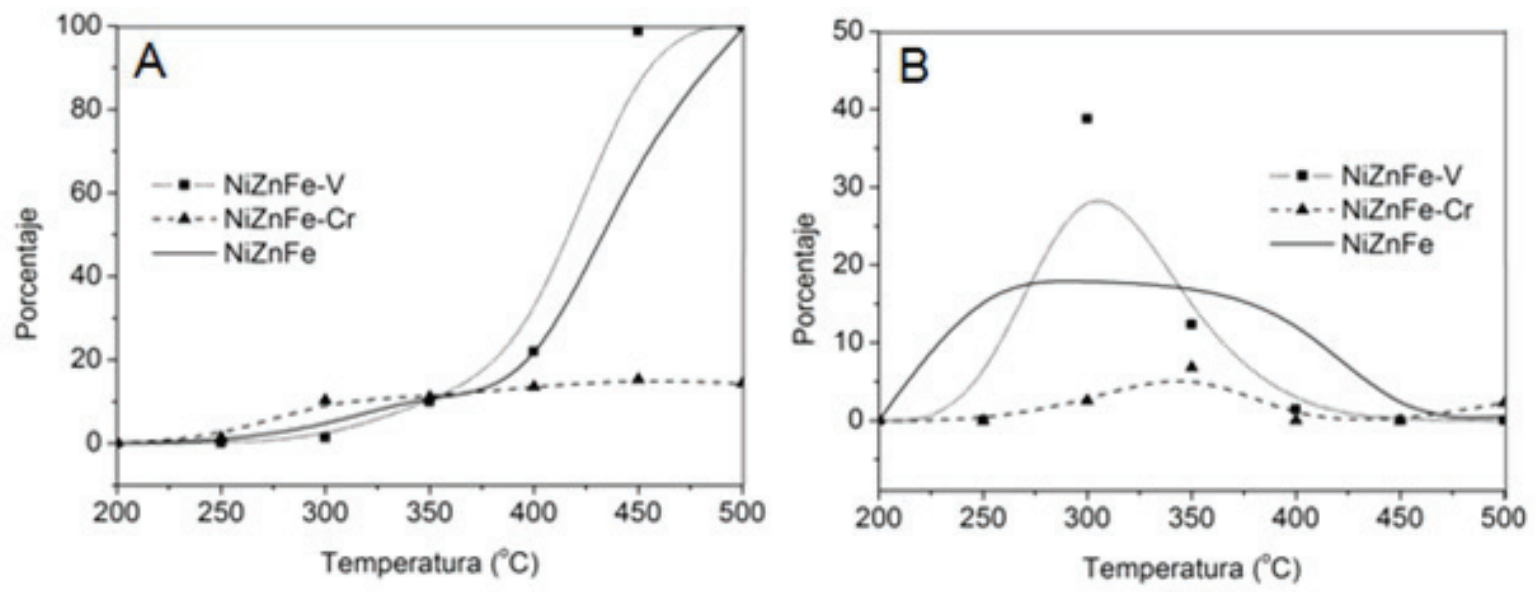

Figura 9. Resultados de (A) Conversión y (B) Selectividad de los materiales en el sistema NiZnFe

\section{Conclusiones}

El método hidrotérmico es un método viable para la obtención de materiales laminares tipo hidrotalcita, siendo posible realizar modificaciones que dan lugar a materiales con composiciones interesantes, como los obtenidos en este trabajo.

A partir de los análisis elementales fue posible evidenciar la incorporación exitosa de los tres metales involucrados en cada material sintetizado; adicionalmente se logró observar la presencia de especies de $\mathrm{V}$ y $\mathrm{Cr}$ en los sólidos. De igual forma y complementando con los análisis térmicos, los resultados experimentales se ajustaron a la fórmula química establecida para materiales tipo hidrotalcita, y de ésta forma fueron propuestas las fórmulas químicas asociadas a los nuevos materiales sintetizados.

Los análisis térmicos dieron cuenta de la incorporación de especies no volátiles en los materiales, evidenciándose una menor pérdida de peso en los sólidos modificados. La diferencia en pérdidas de peso fue mucho más marcada en el sistema NiZnFe, especialmente el material modificado con $\mathrm{Cr}$.

La presencia de $\mathrm{V}$ en el sistema $\mathrm{NiMgFe}$ favoreció los resultados de selectividad hacia el propeno a $300^{\circ} \mathrm{C}$, sin embargo, por encima de esta temperatura dicho valor se vio reducido con respecto al material sin modificar, donde esta disminución se presentó a mayor temperatura.

\section{Agradecimientos}

A la Universidad de Antioquia por el apoyo financiero al proyecto a través del CODI y sostenibilidad, y a Colciencias por el apoyo financiero a través del programa de becas doctorales.

\section{Referencias Bibliográficas}

Auerbach, M., Carrado, K.A., \& Dutta, P.K. (2004). Handbook of layered materials. New York: Marcel Dekker.

Bulánek, R., Kaluzová, A., Setnicka, M., Zukal, A., Cicmanec, P., \& Mayerova, J. (2012). Study of vanadium based mesoporous silicas for oxidative dehydrogenation of propane and n-butane. Catalysis Today 179, 149-158.

Caballero L. M. (2002). Introducción a la catálisis heterogénea. Bogotá: Universidad Nacional de Colombia, Departamento de Ingeniería Química.

Cavani, F., Trifirò, F., Vaccari,A. (1991). Hydrotalcite-type anionic clays: preparation, properties and applications. Catalysis Today 11 (2), 173-301.

Cavani, F., Ballarini, N., Cericola, A. (2007). Oxidative dehydrogenation of ethane and propane: how far from commercial implementation. Catalysis Today 127 (1-4), 113-131. 
Crivello, M., Pérez, C., Herrero, E., Ghione, G., Casuscelli, S., \& Rodríguez-Castellón, E. (2005). Characterization of Al-Cu and Al-Cu$\mathrm{Mg}$ mixed oxides and their catalytic activity in dehydrogenation of 2-octanol. Catalysis Today 107-108, 215-222.

Dula, R., Wciso, K., Stoch, J., Grzybowska, B., Serwicka, E.M., Kooli, F., Bahranowski, K., \& Gawe, A. (2002). Layered double hydroxidederived vanadium catalysts for oxidative dehydrogenation of propane Influence of interlayer-doping versus layer-doping. Applied Catalysis A: General 230 (1-2), 281-291.

Gabriele C., Siglinda P. (2008). Catalysis by layered materials: A review. Microporous and mesoporous 107 (1-2), 3-15.

Kovanda, F., Kolousek, D., Cílová, Z., \& Hulínský, V. (2005). Crystallization of synthetic hydrotalcite under hidrotermal conditions. Applied clay science 28 (1-4), 101-109.

Malherbe, F., Forano, C., Sharma, B., Atkins, M.P., \& Besse, J.P. (1998). Hydrotalcite-like compounds as precursor for mixed oxides catalysts in the oxydehydrogenation of ethylbenzene. Applied Clay Science 13, 381399.

Mitchell, P. C. H., \& Wass, S. A. (2005). Propane dehydrogenation over molybdenum hydrotalcite catalysts. Applied catalysis A: General 225 (1-2), 153-165.

Mitran, G., Cacciaguerra, G., Loridant, S., Tichit, D., Marcu, J - C., (2012). Oxidative dehydrogenation of propane over cobaltcontaining mixed oxides obtained from LDH precursors. Applied Catalysis A: General. (417418). 153-162.

Rives, V., Labajos, F.M., Trujillano, R., Romeo, E., Royo, C., \& Monzon, A. (1998). Acetylene hydrogenation on $\mathrm{Ni}-\mathrm{Al}-\mathrm{Cr}$ oxide catalysts: the role of added Zn. Applied Clay Science 13 (5-6), 363-379.

Valverde, J., Echavarría, A., Ribeiro, M.F., Palacio, L.A., \& Eon, J.G. (2012). Decavanadateintercalated $\mathrm{Ni}-\mathrm{Al}$ hydrotalcites as precursor of mixed oxides for the oxidative dehydrogenation of propane. Catalysis Today. 192 (1, 36-43.

Wang, L., Chu, W., Jiang, C., Liu, Y., Wen, J., \& Xie, Z. (2012). Oxidative dehydrogenation of propane over Ni-Mo-Mg-O catalysis. Journal of Natural Gas Chemistry 21, 43-48.

Ye, X., Ma, N., Hua, W., Yue, Y.,Miao, C., Xie, Z., \& Gao, Z. (2004). Dehydrogenation of ethylbenzene in the presence of $\mathrm{CO}_{2}$ over catalysts prepared from hydrotalcite-like precursors. Journal of molecular catalysis A:chemical 217 (1-2), 103-108. 